\title{
Anterior Chamber Changes After Implantable Collamer Lens Implantation in High Myopia Using Pentacam: A Prospective Study
}

\author{
Mohamed Nagy Elmohamady $\cdot$ Walid Abdelghaffar
}

Received: August 12, 2017 / Published online: September 20, 2017

(c) The Author(s) 2017. This article is an open access publication

\begin{abstract}
Introduction: The objective of this study was to evaluate the changes of the anterior chamber (AC) of the eye after implantable Collamer lens (ICL) implantation in high myopia by using the Pentacam.

Methods: The prospective study included 34 high myopic patients (34 eyes). All patients were treated with Visian implantable Collamer lens (ICL) (Staar Surgical Co.) implantation. The Pentacam (Oculus) was used for all cases preoperatively and 1, 3 and 6 months postoperatively. AC angle (ACA), AC volume (ACV), central AC depth (CACD), ICL vault, central corneal thickness (CCT), pupil diameter (PD), $K$ readings and intraocular pressure (IOP) were recorded.
\end{abstract}

Results: Our study included 34 eyes of 34 patients, 20 females (59\%) and 14 males (41\%). Mean ACA was $36.93^{\circ} \pm 4.75^{\circ}$ preoperatively, $25.17^{\circ} \pm 5.64^{\circ}$ and $25.23^{\circ} \pm 6.05^{\circ} 1$ and 3 months postoperatively $(p<0.001)$. Mean ACV was $191.24 \pm 11.18 \mathrm{~mm}^{3}$ preoperatively, $141.53 \pm 16.77$ and $142.11 \pm 15.89 \mathrm{~mm}^{3} 1$ and

Enhanced content To view enhanced content for this article go to http://www.medengine.com/Redeem/ 323CF06029964829.

M. N. Elmohamady $(\varangle) \cdot$ W. Abdelghaffar Ophthalmology Department, Faculty of Medicine, Benha University, Benha, Qaliopia, Egypt e-mail: mohamed.saad@fmed.bu.edu.eg
3 months postoperatively $(p<0.001)$. Mean CACD was $3.59 \pm 0.17 \mathrm{~mm}$ preoperatively, $2.83 \pm 0.24 \mathrm{~mm}$ and $2.96 \pm 0.25 \mathrm{~mm} 1$ and 3 months postoperatively $(p<0.001)$. The ICL vault decreased from $0.556 \pm 0.033 \mathrm{~mm}$ 1 month postoperatively to $0.431 \pm 0.056 \mathrm{~mm}$ 3 months postoperatively $(p<0.001)$. There were no statistically significant differences for any of the AC parameters among measurements taken 3, 6 and 12 months postoperatively. There were no statistically significant differences between preoperative and any postoperative measurements of CCT, PD and $K$ readings. Conclusions: ICL implantation for correction of high myopia leads to significant changes in the anterior chamber angle, volume and depth and insignificant changes in the pupil diameter, CCT and $K$ readings.

Keywords: Anterior chamber; Collamer, myopia; ICL; Pentacam

\section{INTRODUCTION}

The Visian Implantable Collamer Lens (ICL, STAAR Surgical, Nidau, Switzerland), a posterior chamber phakic intraocular lens (IOL), has been widely used and accepted for the correction of high myopia [1]. It has been reported to be a safe, effective and reversible approach but patients must be aware of possible complications, such as lens opacification, endothelial 
cell loss, increased intraocular pressure (IOP), inflammation and others $[2,3]$.

The anterior segment parameters are vital to choose the suitable refractive surgery. Central anterior chamber depth (CACD) and white to white (WTW) are important parameters for evaluating eligibility for ICL surgery and selecting the proper size of ICL [4]. A CACD value less than $2.80 \mathrm{~mm}$ is a contraindication to ICL surgery. The central vault, the distance between the back surface of the ICL and the front surface of the lens, is of value for estimating the safety of surgery postoperatively. An excessive vault may lead to glaucoma, and an insufficient vault increases the risk of anterior subcapsular cataracts [5]. Hence, an accurate and objective CACD and central vault assessment are both of great value for successful ICL surgery.

The Pentacam generates a three-dimensional virtual model of the anterior segment in about $2 \mathrm{~s}$. Software allows evaluation and quantification of anterior segment parameters such as the central anterior chamber depth (CACD), anterior chamber volume (ACV), pupil diameter (PD) and anterior chamber angle (ACA) of cross-section photographs from $0^{\circ}$ to $360^{\circ}$ [6].

Previous studies have been done to evaluate the safety and efficacy of ICL as a refractive treatment $[1,2,7]$, to compare different methods of anterior segment imaging postoperatively [8] and to detect changes in anterior segment parameters after surgery [9]. However, none of these studies compared the pre- and postoperative anterior segment parameters using the Pentacam. The aim of our work is to evaluate changes of the anterior chamber (AC) of the eye after ICL implantation in high myopia using the Pentacam.

\section{METHODS}

Thirty-four eyes of 34 patients (20 females, 14 males) who underwent ICL implantation for correction of high myopia at the Ophthalmology Department of Benha University Hospital between January 2015 and March 2016 were included. Patients with unrealistic expectations; anterior chamber depth less than $2.80 \mathrm{~mm}$; IOP greater than $20 \mathrm{mmHg}$; a history of ocular surgery or trauma, cataract, glaucoma, diabetes mellitus, other ocular or systemic autoimmune diseases; and age less than 18 years were excluded from the study. The study was agreed upon by the research ethics committee of the Benha Faculty of Medicine. Informed consent was signed by all participants as per the Declaration of Helsinki.

\section{Clinical Evaluation}

We did a full ophthalmic examination for all subjects before the operation and 1, 3, 6 and 12 months postoperatively. The examinations included uncorrected and corrected distance visual acuity (UDVA and CDVA) according to the Snellen chart, manifest and cycloplegic refractions, slit-lamp bio-microscopy, fundus examinations and intraocular pressure.

\section{Imaging}

Imaging of the anterior segment preoperatively and 1, 3, 6 and 12 months after surgery using a rotating Scheimpflug camera (Pentacam version 1.20 , Oculus, Germany) was carried out by the same observer and under scotopic conditions, with the patient's chin placed on the chin rest and the forehead against the forehead strap. The patient was asked to fixate on the fixation target. Once alignment was perfect, 25 Scheimpflug images were automatically recorded within $2 \mathrm{~s}$. Image quality was checked using the quality factor value for each eye. The AC angle (ACA) was measured superiorly, inferiorly, nasally and temporally, and then a mean ACA was calculated; the AC volume (ACV), central AC depth (CACD) and peripheral AC depth (PACD) were measured $4 \mathrm{~mm}$ from the center superiorly, inferiorly, nasally and temporally. Then, a mean PACD was calculated, and central corneal thickness (CCT), pupil diameter (PD) and $K$ readings were recorded. The central vault of the ICL was measured 1, 3, 6 and 12 months after surgery using the image analysis program accompanying the device. 


\section{Implantable Collamer Lens Calculation}

The ICL is $6.0 \mathrm{~mm}$ wide, and four sizes are available $(11.5,12.0,12.5$ and $13.0 \mathrm{~mm}$ in from -3.00 to $-23.00 \mathrm{DS})$. Calculations of ICL power and size were done by the manufacturer. A modified vertex formula was used in power calculations. The variables in the formula are preoperative manifest and cycloplegic refractions, $K$ readings, CCT and central ACD. The size of the ICL was determined by the STAAR sizing formula based on the WTW and CACD obtained by the Pentacam. For CACD of 2.80-3.00 mm, ICL length $=\mathrm{WTW}+0.2-0.4-$ $\mathrm{mm}$; for CACD of $3.00-3.50 \mathrm{~mm}$, ICL length $=\mathrm{WTW}+0.4-0.6 \mathrm{~mm}$; for $\mathrm{CACD}$ of $3.50-3.70 \mathrm{~mm}, \quad$ ICL $\quad$ length $=$ WTW $+0.6-$ $0.8 \mathrm{~mm}$.

\section{Surgical Procedure}

Two YAG peripheral iridectomies were done at two different sites 2 weeks before surgery. Mydriatic agents were instilled four times at 10-min intervals before surgery. After topical anesthesia, a 3-mm temporal corneal incision was created. Viscoelastic material was injected in the AC. An injector cartridge (STAAR Surgical) was used to insert the ICL V4 without hole. The four footplates of the ICL were placed on the ciliary sulcus along the $180^{\circ}$ axis. Viscoelastic material was completely removed using buffered salt solution. No astigmatic keratotomy or limbal relaxing incision was made. Postoperatively, tobramycin $0.3 \%$-dexamethasone $0.1 \%$ (Tobradex) and moxifloxacin $0.5 \%$ (Vigamox) eye drops were administered topically three times daily for 2 weeks.

\section{Statistical Analysis}

Statistical analysis was done using SPSS version 20.0 (SPSS Inc., IBM, USA), and the results are expressed as mean \pm standard deviation (SD). The Kolmogorov-Smirnov test was employed to assure the normal distribution of the data. Comparison between pre- and postoperative measurements was done using independent $t$ tests. A $p$ value $\leq 0.05$ was considered statistically significant. Spearman's rho test was used to measure the strength of association between variables.

\section{RESULTS}

This study included 34 eyes of 34 patients, 20 females (59\%) and 8 males (41\%). Mean age was $24.76 \pm 5.14$ (range, 19-37) years. Mean spherical equivalent (SE) of refractive error was $-16.91 \pm 2.37 \mathrm{D}$ before surgery. One month after surgery, SE was $-0.46 \pm 0.37 \mathrm{D}$, and 3 months postoperatively SE became $-0.51 \pm 0.32 \mathrm{D}(p=0.553)$ (Table 1$)$. No intraor postoperative complications occurred except for increased IOP ( $>21 \mathrm{mmHg}$ ) in five eyes, which was managed by medical treatment with no need for surgical intervention.

One month after surgery, ACV, ACA, CACD and PACD decreased significantly $(p<0.001)$. On the other hand, PD, CCT, keratometric values (K1 and $K 2)$ and IOP showed insignificant change with $p$ values of 0.062 (PD was near to significance), 0.436, 0.145, 0.477 and 0.207, respectively, as shown in Table 2 .

Table 1 Patient demographics: selected pre- and postoperative characteristics

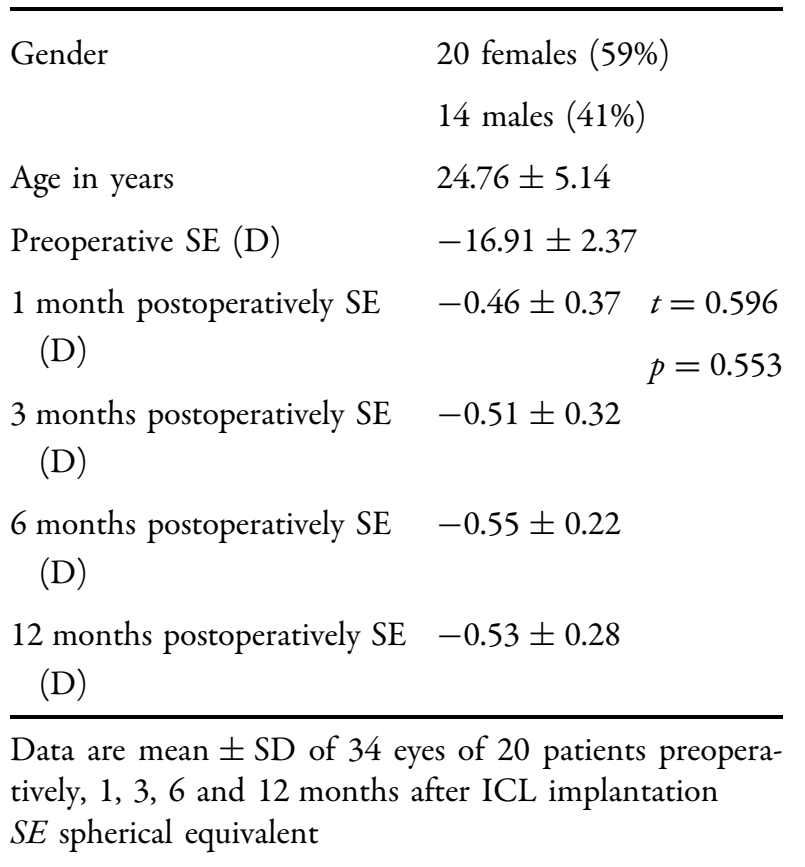


Table 2 Changes in anterior chamber parameters 1 month after ICL implantation

\begin{tabular}{|c|c|c|c|c|c|}
\hline Parameters & Preoperative & $\begin{array}{l}1 \text { Month } \\
\text { postoperative }\end{array}$ & $\begin{array}{l}3 \text { Months } \\
\text { postoperative }\end{array}$ & $\begin{array}{l}6 \text { Months } \\
\text { postoperative }\end{array}$ & $\begin{array}{l}12 \text { Months } \\
\text { postoperative }\end{array}$ \\
\hline $\begin{array}{l}\mathrm{ACV} \\
\quad\left(\mathrm{mm}^{3}\right)\end{array}$ & $191.24 \pm 11.18$ & $141.53 \pm 16.77^{*}$ & $142.11 \pm 15.89^{*}$ & $142.05 \pm 14.76^{*}$ & $142.07 \pm 16.62^{*}$ \\
\hline $\operatorname{ACA}\left({ }^{\circ}\right)$ & $36.93 \pm 4.75$ & $25.17 \pm 5.64^{*}$ & $25.23 \pm 6.05^{*}$ & $25.30 \pm 5.14^{*}$ & $25.32 \pm 7.24^{*}$ \\
\hline $\begin{array}{l}\text { CACD } \\
(\mathrm{mm})\end{array}$ & $3.59 \pm 0.17$ & $2.83 \pm 0.24^{*}$ & $2.96 \pm 0.25^{* \neq}$ & $2.94 \pm 0.36^{* \neq}$ & $2.95 \pm 0.31^{* \neq}$ \\
\hline $\begin{array}{l}\text { PACD } \\
(\mathrm{mm})\end{array}$ & $2.78 \pm 0.21$ & $2.13 \pm 0.19^{*}$ & $2.19 \pm 0.13^{*}$ & $2.12 \pm 0.17^{*}$ & $2.15 \pm 0.20^{*}$ \\
\hline PD (mm) & $5.11 \pm 0.56$ & $4.77 \pm 0.88$ & $4.79 \pm 0.90$ & $4.75 \pm 1.04$ & $4.82 \pm 1.17$ \\
\hline $\operatorname{CCT}(\mu \mathrm{m})$ & $500.39 \pm 19.71$ & $504.16 \pm 20.12$ & $501.16 \pm 19.52$ & $503.06 \pm 22.14$ & $502.06 \pm 20.86$ \\
\hline$K 1(\mathrm{D})$ & $43.28 \pm 1.11$ & $42.89 \pm 1.07$ & $42.96 \pm 1.15$ & $42.88 \pm 1.31$ & $42.74 \pm 1.39$ \\
\hline$K 2(\mathrm{D})$ & $44.65 \pm 1.34$ & $44.42 \pm 1.31$ & $44.35 \pm 1.28$ & $44.58 \pm 1.40$ & $44.47 \pm 1.44$ \\
\hline $\begin{array}{l}\text { IOP } \\
\qquad(\mathrm{mmHg})\end{array}$ & $11.33 \pm 2.41$ & $12.19 \pm 3.11$ & $12.12 \pm 2.92$ & $12.69 \pm 2.64$ & $12.43 \pm 3.57$ \\
\hline Vault (mm) & - & $0.556 \pm 0.033$ & $0.431 \pm 0.056 \neq$ & $0.429 \pm 0.049 \neq$ & $0.426 \pm 0.064 \neq$ \\
\hline
\end{tabular}

Data are mean \pm SD of 34 eyes preoperatively, 1, 3, 6 and 12 months after ICL implantation

$A C V$ anterior chamber volume, $A C A$ anterior chamber angle, $C A C D$ central anterior chamber depth, $P A C D$ peripheral anterior chamber depth, $P D$ pupil diameter, $C C T$ central corneal thickness, IOP intraocular pressure

* Statistically significant difference from preoperative value, ${ }^{\neq}$statistically significant difference from 1 month postoperative value

Spearman's rho test was used to measure the correlation between the central ICL vault and CACD at 1 month postoperatively; we found a statistically significant negative correlation (Spearman's correlation coefficient $r=-0.8224$, $p<0.001)$. We also found a statistically significant positive correlation between the central ICL vault and PD at 1 month postoperatively (Spearman's correlation coefficient $r=0.9429$, $p<0.001)$.

At the 3-month visit, the ACV, ACA, CACD and PACD had decreased significantly from the preoperative values $(p<0.001)$, while $\mathrm{PD}, \mathrm{CCT}$, keratometric values ( $K 1$ and $K 2)$ and IOP showed insignificant changes with $p$ values of $0.083,0.872,0.274,0.349$ and 0.228 , respectively (Table 2 ).

There were no statistically significant changes in AC parameters between 1 and 3 months after ICL implantation except for the ICL vault, which decreased from $0.556 \pm 0.033 \mathrm{~mm}$ 1 month post-surgery to $0.431 \pm 0.056 \mathrm{~mm}$ 3 months postoperatively $(p<0.0001)$, and CACD, which increased from $2.83 \pm 0.24 \mathrm{~mm} 1$ to $2.96 \pm 0.253$ months postoperatively with $p=0.0323$ (Table 2 ). We found no statistically significant changes in AC parameters among the 3-, 6- and 12-month results after ICL implantation as all $p$ values were $>0.05$ (Table 2).

\section{DISCUSSION}

The implantation of ICL has been shown to be an effective surgical treatment for the correction of refractive errors, offering good optical quality. With expanding use of ICLs worldwide, several studies addressing their efficacy and safety have been done and are still of current interest $[1,2,6,10-13]$. Our study aimed to 
evaluate AC changes after ICL implantation using the Pentacam. Our results pointed to significant changes in ACA, ACV, CACD and PACD 1, 3, 6 and 12 months after surgery as compared to the preoperative parameters. On the other hand, we found insignificant changes in CCT, $K$ readings, PD and IOP.

We found that ACA decreased by $31.8 \%$, $31.6 \%, 31.5 \%$ and $31.4 \%$ at $1,3,6$ and 12 months postoperatively, respectively. ACA changed from $36.93^{\circ} \pm 4.75^{\circ}$ preoperatively to $25.17^{\circ} \pm 5.61^{\circ} 1$ month postoperatively to $25.23^{\circ} \pm 6.05^{\circ} 3$ months postoperatively and to $25.3^{\circ} \pm 5.14^{\circ} 6$ months postoperatively. These results were the same as those of Chung and associates [10] who detected a $31.7 \%$ reduction in the trabecular-iris angle (from $38.1^{\circ} \pm 8.7^{\circ}$ to $26.0^{\circ} \pm 6.5^{\circ}$ ) using UBM (ultrasound bio-microscopy) 1 month after surgery. FernándezVigo and associates [11] found an angle reduction of $34.5 \% 3$ months after surgery using Fourier-domain optical coherence tomography, and they suggested that this reduction was caused by the convex shape of the ICL and not due to disturbed aqueous flow. In our study, there were no statistically significant differences between ACAs at 1 and 3 months postoperatively $(p=0.966)$. Thus, the initial narrowing of the ACA remained stable for 3 months post-surgery. Consistently, this was the case in Fernández-Vigo and associates' study [11] with no significant change in 3 months and in Chung and associates study [10] as there were no significant changes in ACA from 1 month postoperatively until the end of follow-up, which was a mean of 33 months.

Our results showed a significant reduction in CACD by about $21.17 \%, 18.11 \%, 18.10 \%$ and $17.83 \%$ at $1,3,6$ and 12 months postoperatively, respectively. This reduction was mainly due to vaulting of the ICL, as the CACD is measured postoperatively from the back surface of the cornea to the anterior surface of the ICL. We found statistically significant negative correlation between the vault and CACD. The central ICL vault in our study was $0.556 \pm 0.033 \mathrm{~mm} 1 \mathrm{month}$ postoperatively and decreased significantly to $0.431 \pm 0.056 \mathrm{~mm}$ 3 months after implantation $(p<0.0001)$; it then decreased insignificantly to $0.429 \pm 0.049$ and $0.426 \pm 0.064 \mathrm{~mm} 6$ and 12 months postoperatively, respectively. These results are in line with results of Ju et al. [12]. They found that mean ACD measured with AS-OCT was $3.28 \pm 0.14 \mathrm{~mm}$ preoperatively and $2.45 \pm 0.22 \mathrm{~mm} 3$ months after surgery. ACD showed a statistically significant reduction of $25.4 \%(p<0.01)$ in their study.

We found that PD insignificantly decreased by $6.7 \%, 6.2 \%, 7 \%$ and $5.7 \%$ at $1,3,6$ and 12 months postoperatively, respectively. Although the change was statistically insignificant, it is worth mentioning that the $p$ value was 0.062 , which means it was close to the significance. This was different from results of previous studies $[13,14]$, where a significant reduction in $\mathrm{PD}$ was observed both 1 and 3 months after ICL implantation. Li and associates [13] attributed this reduction to the contact between the iris and ICL, which limits postoperative pupil movements, as they found a significant correlation between lower degrees of myopia and decreased size of the pupil. Our results were different perhaps because our patients had higher degrees of myopia as mean SE was $-16.91 \pm 2.37 \mathrm{D}$ in our study, while it was $-12.50 \pm 4.28$ in their study. We also found a statistically significant positive correlation between the vault and PD. This was also found by Chen et al. [9] who stated that pupil size is a vital factor in vault change, with an increasing vault observed postoperatively from 1 day to 1 week associated with the lessening effects of pharmacological miosis and increasing pupil size.

IOP was elevated $>21 \mathrm{mmHg}$ in $14.7 \%$ (5 eyes) of our cases, with three eyes showed the increased IOP during the first week, which can be related to retained viscoelastic material, while two eyes had high IOP after 1 month; this may have been due to a steroid-induced response. This was nearly the case in Almalki and colleagues' study [15]; they found elevated IOP in $10.8 \%$ of cases. CCT showed a nonsignificant change in our cases; this was because of proper IOP control and minimal or no surgical trauma to the endothelium. Also, $K$ readings suffered from insignificant changes because the incision was small in size $(3 \mathrm{~mm})$ and temporal in site. 
Our study was limited by the relatively small number of subjects. The follow-up period was 12 months, but some changes may continue to evolve later. We had no chance to use another imaging technique besides the Pentacam to compare their results.

\section{CONCLUSIONS}

Our results support the view that ICL implantation is a safe and effective option for treatment of high myopia and that the Pentacam can be used to evaluate anterior segment parameters. ICL implantation reduces the ACA and CACD significantly. These changes should be considered during evaluation of case eligibility for ICL implantation. ICL implantation has an insignificant effect on pupil size and corneal curvature, which may guarantee good quality of vision after surgery.

\section{ACKNOWLEDGEMENTS}

No funding or sponsorship was received for this study or publication of this article. The article processing charges were funded by the authors. Both authors meet the International Committee of Medical Journal Editors (ICMJE) criteria for authorship for this manuscript, take responsibility for the integrity of the work and have given final approval for the version to be published. Part of this study was presented as a poster at the ESCRS conference, Copenhagen, September 2016.

Disclosures. M. N. Elmohamady and W. Abdelghaffar have nothing to disclose.

Compliance with Ethics Guidelines. All procedures followed were in accordance with the ethical standards of the responsible committee on human experimentation at Benha University and with the Helsinki Declaration of 1964, as revised in 2013. Informed consent was obtained from all participants.

Data Availability. The data sets during and/ or analyzed during the current study are available from the corresponding author on reasonable request.

Open Access. This article is distributed under the terms of the Creative Commons Attribution-NonCommercial 4.0 International License (http://creativecommons.org/licenses/ by-nc/4.0/), which permits any noncommercial use, distribution, and reproduction in any medium, provided you give appropriate credit to the original author(s) and the source, provide a link to the Creative Commons license, and indicate if changes were made.

\section{REFERENCES}

1. Kamiya K, Shimizu K, Igarashi A, Hikita F, Komatsu M. Four-year follow-up of implantable collamer lens implantation for moderate to high myopia. Arch Ophthalmol. 2009;127:845-50.

2. Igarashi A, Shimizu K, Kamiya K. Eight-year follow-up of posterior chamber phakic intraocular lens implantation for moderate to high myopia. Am J Ophthalmol. 2014;157(3):532-9.

3. Sari ES, Pinero DP, Kubaloglu A, Evcili PS, Koytak A, Kutluturk I, Ozerturk Y. Toric implantable collamer lens for moderate to high myopic astigmatism: 3 -year follow-up. Graefes Arch Clin Exp Ophthalmol. 2013;251(5):1413-22.

4. Lim DH, Lee MG, Chung ES, Chung TY. Clinical results of posterior chamber phakic intraocular lens implantation in eyes with low anterior chamber depth. Am J Ophthalmol. 2014;158(3):447-54.

5. Fernandes P, González-Méijome JM, Madrid-Costa D, Ferrer-Blasco T, Jorge J, Montés-Micó R. Implantable collamer posterior chamber intraocular lenses: a review of potential complications. J Refract Surg. 2011;27(10):765-76.

6. Esmaeili A, Barazandeh B, Ahmadi S, Haghi A, Ahmadi Hosseini SM, Abolbashari F. Assessment of the anterior chamber parameters after laser iridotomy in primary angle close suspect using Pentacam and gonioscopy. Int J Ophthalmol. 2013;6:680-4.

7. Ju Y, Gao X-W, Ren B. Posterior chamber phakic intraocular lens implantation for high myopia. Int J Ophthalmol. 2013;6(6):831-5. doi:10.3980/j.issn. 2222-3959.2013.06.16.

8. Zhang J, Luo H, Zhang J, Yu K. Comparison of anterior section parameters using anterior segment 
optical coherence tomography and ultrasound biomicroscopy in myopic patients after ICL implantation. Int J Ophthalmol. 2016;9(1):58-62.

9. Chen X, Miao H, Naidu RK, Wang X, Zhou X. Comparison of early changes in and factors affecting vault following posterior chamber phakic implantable collamer lens implantation without and with a central hole (ICL V4 and ICL V4c). BMC Ophthalmol. 2016;16(1):161 (PMC. Web. 29 Sept. 2016).

10. Chung SC, Park MO, Lee K, Ahn ES. Chung. Changes in iridocorneal angle structure and trabecular pigmentation with STAAR implantable collamer lens during 2 years. J Refract Surg. 2009;25(3):251-8.

11. Fernández-Vigo JI, Macarro-Merino A, FernándezVigo C, et al. Effects of implantable Collamer lens V4c placement on iridocorneal angle measurements by Fourier-domain optical coherence tomography. Am J Ophthalmol. 2016;162(4352):e1.
12. Ju Y, Gao X-W, Ren B. Posterior chamber phakic intraocular lens implantation for high myopia. Int J Ophthalmol. 2013;6(6):831-5 (PMC. Web. 21 Nov. 2016).

13. Li D, Yang Y, Su C, Yin H, Liu X. Pupil diameter changes in high myopes after collamer lens implantation. Optom Vis Sci. 2015;92(12):1161-9. doi:10.1097/OPX.0000000000000723.

14. Chun YS, Park IK, Lee HI, Lee JH, Kim JC. Iris and trabecular meshwork pigment changes after posterior chamber phakic intraocular lens implantation. J Cataract Refract Surg. 2006;32:1452-8.

15. Almalki S, Abubaker A, Alsabaani NA, Edward DP. Causes of elevated intraocular pressure following implantation of phakic intraocular lenses for myopia. Int Ophthalmol. 2016;36:259-65. doi:10.1007/ s10792-015-0112-4. 